\title{
STRESSES AROUND IMPLANTS AND NATURAL ABUTMENTS IN FREE END RPD
}

\author{
Emad M.T. Agamy* and Gehan F. Mohamed**
}

\begin{abstract}
Aim The aim of the present study is to evaluate the stresses created in the supporting structures of implant supported free end saddle RPD retained by extra-coronal attachment (periodontal ligament of abutment teeth and bone implant interface) either with or without splinting of the abutment teeth.

Materials and methods: The model represented a case of mandibular Kennedy class I partially edentulous situation. The last standing natural abutment was the $1^{\text {st }}$ premolar bilaterally. An implant was drawn to support the simulated RPD at the area of $2^{\text {nd }}$ molar. An extra-coronal attachment was used to retain the RPD to the natural abutment (s). The stress distributions were evaluated using a three-dimensional Finite element analysis (FEA).
\end{abstract}

Results: The stresses generated in the periodontal ligament of the abutment teeth were affected by splinting. Placement of an implant in the distal area to support a RPD decreases the stresses exerted by the denture to the abutments and supporting tissues. The effect of implant placement is more important in cases of non-splinted model as it shares greater stresses than splinted designs.

Conclusion: Splinting the main abutment to the adjacent tooth might be beneficial as it produces more favorable stress distribution and can tolerate non axial loading.

\section{INTRODUCTION}

Removable partial dentures with tooth and mucosal support are devices of great complexity that; despite recent technological methods of their construction, constitute a challenge to the professionals who need their use. This double system of support causes the occurrence of unequal stresses around the abutment teeth that increases the possibility of bone resorption. It also results in rotation of the distal extension removable partial denture around its most posterior abutment to induce heavy stresses and excessive bone resorption of the underlying residual ridge.

Takayuki and Noriyuki supposed that loss of molar support in free end saddle cases causes increasing stress and strain in the periodontal

\footnotetext{
* Assoc Prof and Head Prosthetic Dentistry Department. Minia University.

** Prof. of Prosthetic Dentistry and Dean; Faculty of Dentistry, Minia University.
} 
structures surrounding the premolars. They recommended that the management of such patients should focus on preservation of the remaining oral structures as well as the long-term survivability of the remaining dentition.

Various solutions were proposed to solve the problems of distal extension RPDs. Among these solutions; the use of attachments, especially the extra-coronal type, is considered more efficient in restoring function, providing retention as well as giving more esthetics appearance ${ }^{3,4}$. However, from the biomechanical point of view; the use of attachments may lead to excessive torque applied to the abutment which may necessitate splinting of more than one abutment. Teeth splinting can decrease both the displacement and stress concentrations ${ }^{5}$.

Implants play an important role in the success of treatment of free end saddle cases. By placing an implant in the distal edentulous area, this could solve the problems associated with support and sometimes retention of distal extension bases especially in mandibular arch as the denture bearing area is limited ${ }^{6}$. As a possible solution to this clinical challenge, an implant placed bilaterally at the distal extension of the denture base will minimize the resultant denture displacement and rotational movement. The main objective of an implant located under the most posteriorly placed molar of the distal extension denture base is to stabilize the RPD in a vertical direction. Implants placed distally (ideally in the area of the second molar) would effectively change the Kennedy Class I or II situation to that of the Class III. This is the ideal situation in which fewer implants are needed to achieve a successful distal extension RPD. If there is insufficient bone in this area, the implant can be placed more medial although this is not an ideal solution ${ }^{7}$.

\section{MATERIALS AND METHODS}

In the present study, a digital model simulating a bilateral mandibular Kennedy class I situation was constructed. The edentulous span extended to include all molars and second premolars on both sides. This model also simulated a partial denture of skeleton design, supported by two implants "one on each side" and retained by an O-T attachment to the natural abutments. Two models were created by duplication, where the only difference was splinting the first premolar and canine on both sides for the second model.

\section{Construction of the three-dimensional model:}

For constructions of this model a volunteer patient was selected to be subjected to mandibular C.T scanning. This patient was male, 32 years old with no amalgam fillings, no developmental abnormalities, no gross defects, no previous surgery and healthy remaining teeth

CT image acquisition was performed in a DICOM format (Digital Imaging Communication on Medicine) using a multi-slice CT scanner at Light speed 16 slice helical mode (General Electric, Easton Turnpike Fairfield, United States) at Minia University Hospital. The following settings were used according to manufactures instructions; $120 \mathrm{KV}, 150 \mathrm{~mA}, 14 \times 14 \mathrm{~cm}$ field of view, $512 \times 512$ matrix, slice thickness of $0.59 \mathrm{~mm}$, no tilting to the maxilla and slight tilting to the mandible (19 degree) ( to avoid superimposition of the maxillary teeth over mandibular teeth). Using image-processing software (Mimics 10.0; Materialise, Leuven, Belgium), the scanned profiles in DICOM format were translated into 3-D models. The mandible was exported to Solidworks software (Solidworks Corporation, Massachusetts, USA) but it was read as surface body. A decision was made to draw the mandible in Solidworks software guided by its coronal cut images (Fig. 1A) in order to obtain full 3D model. CT cuts were saved as JPEG extension files by screen shots of all processed images to gain 94 photos of $0.59 \mathrm{~mm}$ interval. The $\mathrm{X}$ and $\mathrm{Y}$ axis of the coronal cut images were fixed to a definite position to standardize the images. The teeth; on the 
other hand were exported directly as (.stl) extension file to Solidworks software.

The body of one half of the main mandibular model was constructed. Twenty four pictures were selected from the previous 94 pictures (Fig. 1B). Twenty four planes were created with $2.5 \mathrm{~mm}$ intervals to obtain the average dimensions of the mandible obtained from calculated 3D model by Materialize MIMICs software. Every two-dimensional sketch was drawn by tracing the imported picture from MIMICs software. The approximate shape of the body of the mandible with the main landmarks - such as mental fossa, submandibular fossa, mental foramen, canine eminence and mandibular foramen - was obtained. The drawn sketches were joined together with the loft property which is a special feature contained in Solidworks software to create a three-dimensional model of one half of the basic mandibular body (Fig. 1C).

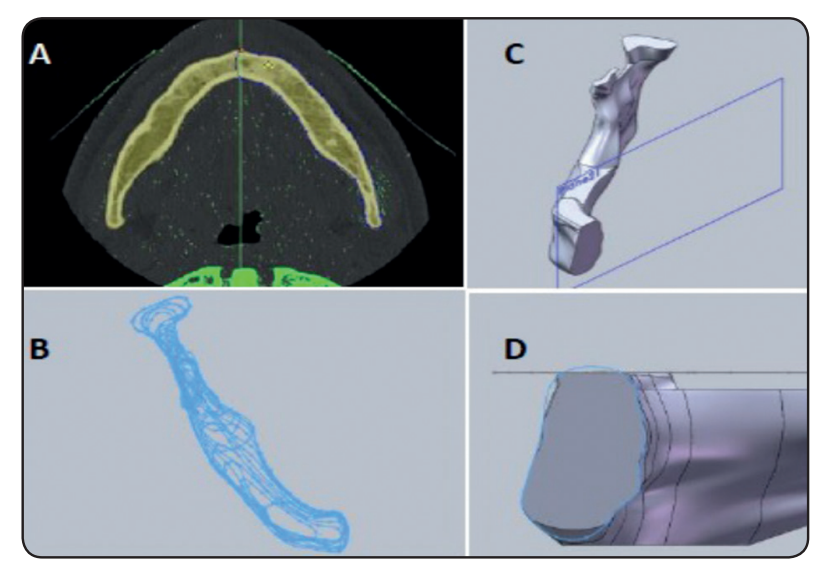

Fig. (1) Importing images to Solidworks and model drawing. A : screen shots from MIMICS; B: Selected planes; C: Created model and D : Modified crest of ridge.

After obtaining the main 3-D model; the crest of the ridge was found to be a flat surface which does not simulate the clinical situation. So the decision was made to draw other sketches on the main model previously drawn to modify the shape of the main model. This modification allows creating a more realistic model (Fig. 1D).
The cancellous bone was drawn with $2 \mathrm{~mm}$ smaller than the original model outline (Fig. 2A) leaving the anterior area purely compact. On the other hand the mucosa was drawn with $2 \mathrm{~mm}$ larger than the original model dimensions (Fig. 2B and C).

The opposite half of the model was generated by mirror imaging of the drawn half through the central plane to acquire the final model (Fig. 2D).

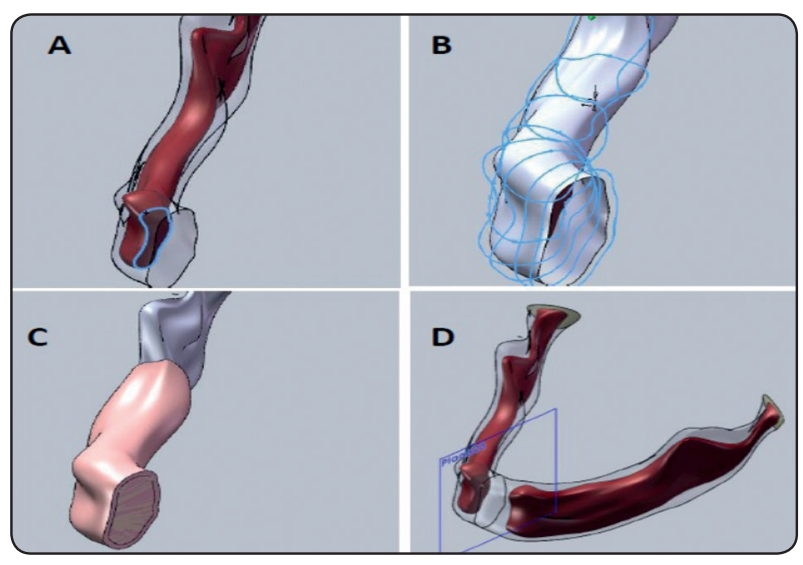

Fig. (2) A: Simulation of cancellous bone, B: simulation of mucosa; C: final model of half mandible; D: Mirror imaging of the other half

The teeth were imported to the solid works software and were read as a solid body. A decision was made to draw the teeth due to the irregular surface of the teeth and the presence of the canal which complicated the meshing process. The root, crown, artificial crown and periodontal ligament were simulated. The teeth with their periodontal ligaments and crowns were arranged in the main model which contains the mucosa, cancellous and compact bone.

Implant and attachments were generated according to the manufacturer's instructions for the product (Screw plant TM, Direct TM implant, The Spectra-System, USA). Metallic framework, acrylic denture base and artificial acrylic teeth were generated. The implant was inserted into its position using the centre of the second molar as a reference point, while the extra-coronal attachment 
was attached to the artificial crown of the main abutment.

All components the model were subtracted from each other leaving one component with reference points by which assembly could be done. This step was repeated for each component independently (Fig 3). All components which are in contact with their mirror structures or with other structures having the same property were combined to each other e.g. compact bone with its mirror.

The constructed components were assembled together to form the two models. The technique of model assembly depended on the mating function of the assembly mode in Solidworks program software. The mating function creates one or more geometrical relationship between different components that will facilitate assembly. The mates used in this model were coincident and parallel. Coincident mate allow coincidence between two edges or surfaces, while parallel mate provide parallelism between two linear edges or flat surfaces.

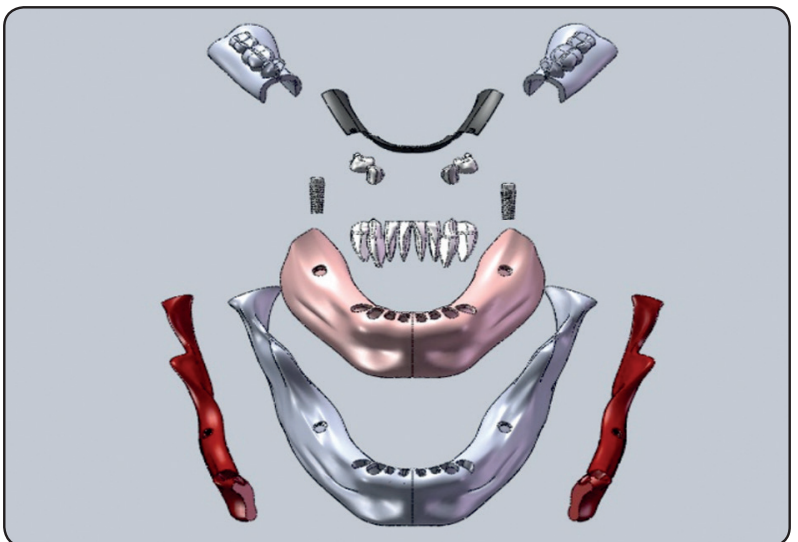

Fig. (3) The final model with its components after subtraction to facilitate mating.

\section{Defining the material properties:}

All materials in the study were considered to be homogenous, isotropic and linearly elastic. The modulus of elasticity and Poisson's ratio for the different component materials used in the study are listed in table (1).
TABLE (1) Materials used in this study and their properties

\begin{tabular}{|c|c|c|c|}
\hline Material & $\mathrm{E} / \mathrm{GPa}$ & $\begin{array}{c}\text { Poisson's } \\
\text { ratio }\end{array}$ & Reference \\
\hline Acrylic Resin & 4.50 & 0.35 & \multirow[t]{5}{*}{ Daas et al ${ }^{8}$} \\
\hline Compact Bone & 13.70 & 0.3 & \\
\hline Cancellous bone & 1.37 & 0.3 & \\
\hline Soft tissue & 0.001 & 0.37 & \\
\hline Ti-6Al-4V & 135 & 0.3 & \\
\hline $\mathrm{Ni}-\mathrm{CR}$ alloy & 188 & 0.28 & \multirow[t]{3}{*}{ Wang et al ${ }^{9}$} \\
\hline Nylon & 2.4 & 0.39 & \\
\hline Dentine & 18.6 & 0.31 & \\
\hline Cr-Co alloy & 218 & 0.33 & Caglaret al ${ }^{10}$ \\
\hline Periodontal ligaments & 0.070 & 0.45 & Geramy et al ${ }^{11}$ \\
\hline
\end{tabular}

\section{Defining contacts and gaps between components}

All components were constructed in a way that assured $100 \%$ contacts along every interface i.e. there were no gaps or interfaces. Two types of contacts were defined:

a) Bonded contact between the two contacting surfaces along the interface; which means that these objects are displaced as one unit upon load application and that the two contacting bodies cannot be separated nor penetrated e.g. compact bone and cancellous.

b) Slip (no penetration) contact between the two contacting surfaces along the interface; which allows some degree of movements between them e.g. mucosa and RPD.

\section{Defining restraints and meshing of the models}

The Restraint property is a special feature in stress analysis programs that allows restriction of displacements on vertices, edges, or faces for use during static analysis of the model. As a solid mesh was planned; the resultant nodes were allowed to translate along any of the 3 orthogonal directions unless a restraint was applied, but no rotation was 
allowed. The restraints applied were fixed restraint on the bottom surface of the mandible and to the mucosa as well as at the condylar area of both sides i.e. no translation was allowed for these surfaces in all directions.

Meshing is the process of subdividing the geometric model into small pieces called elements connected at common points called nodes. A high quality solid mesh was used in this study to create 3D parabolic tetrahedral solid elements. As a solid mesh was used; the resultant nodes were allowed to translate along any of the 3 orthogonal directions unless a restraint was applied, but no rotation was allowed.

\section{Defining loads applied on each model:}

For each model two different loads were applied; each on a separate study:

Vertical loading: Unilateral vertical load of $150 \mathrm{~N}$ distributed on the central fossae of right $2 \mathrm{nd}$ premolar, 1st and 2nd molars.

Oblique loading: Unilateral oblique load of 150 $\mathrm{N}$ distributed on the lingual inclines of the buccal cusps of the right 2nd premolar, 1st and 2nd molars.

\section{Running the analysis}

The analysis was run by the (FFE plus solver) which is an iterative method. Iterative methods solve the equations using approximate techniques; where in each iteration a solution is assumed and the associated errors are evaluated.

\section{Collection of results}

As the analysis was finished; the result tree was activated. The von Mises stress (combined axial and shear total stress) was calculated at the elements in Mega Pascal (Mpa). The "list selected" property was used to calculate the average stresses, the minimum stresses and the maximum stresses values for the elements. This process was carried out for the two loading conditions.

\section{RESULTS}

\section{For loaded side}

Generally, there were higher stress values for the non-splinted model under the vertical load than the splinted. On the other hand; oblique loading generated the highest stresses to the implant of the splinted model followed by the PL of natural abutments of the non-splinted model. (Tables 2 and 3 and figures 4-6)

TABLE (2) Stress values of loaded side implants and periodontal ligaments of 1st premolar (PL/PM) and canine (PL/C) in MPa for both models under vertical loads.

\begin{tabular}{|c|c|c|c|c|c|c|}
\hline & \multicolumn{3}{|c|}{ Model I(non splinted) } & \multicolumn{3}{c|}{ Model II(splinted) } \\
\cline { 2 - 7 } & Implant & PL/PM & PL/C & Implant & PL/PM & PL/C \\
\hline Maximum & 91.045 & 69.218 & 27.78 & 51.467 & 19.411 & $\mathbf{1 0 . 3 5}$ \\
\hline Minimum & 0.002038 & 0.037533 & 0.006876 & 0.00 & 0.030707 & $\mathbf{0 . 0 0 8 6 3 4 1}$ \\
\hline
\end{tabular}

TABLE (3) Stress values of loaded side implants and periodontal ligaments of 1st premolar (PL/PM) and canine (PL/C) in MPa for both models under oblique loads.

\begin{tabular}{|c|c|c|c|c|c|c|}
\hline \multirow{2}{*}{} & \multicolumn{3}{|c|}{ Model I(non splinted) } & \multicolumn{3}{c|}{ Model II(splinted) } \\
\cline { 2 - 8 } & Implant & PL/PM & PL/C & Implant & PL/PM & PL/C \\
\hline Maximum & 129.75 & 186.58 & 28.106 & 143.05 & 27.449 & $\mathbf{2 0 . 3 7 2}$ \\
\hline Minimum & 0.0047254 & 0.58232 & 0.005355 & 0.0061577 & 0.054516 & $\mathbf{0 . 0 1 8 0 4}$ \\
\hline
\end{tabular}




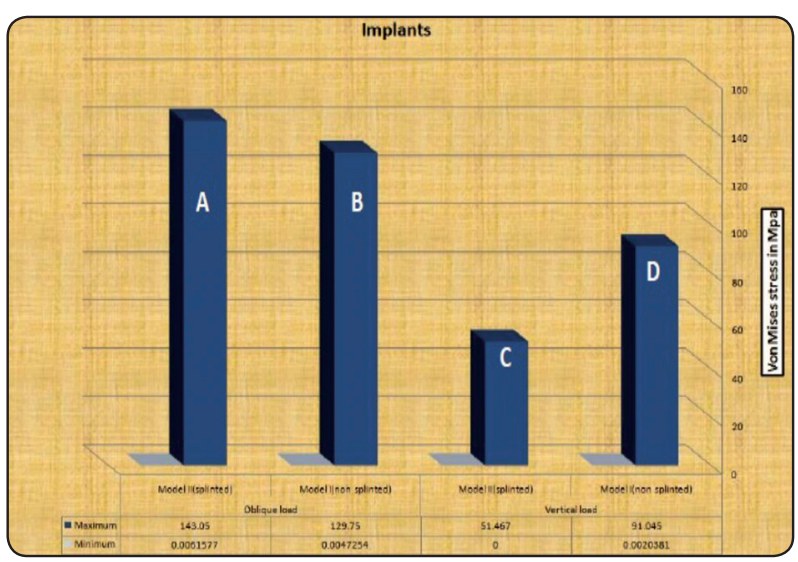

Fig. (4) Bar chart showing maximum stresses around the loaded side implant in MPa. Oblique loading: A; splinted, B; non-splinted. Vertical loading: C; splinted and D nonsplinted.

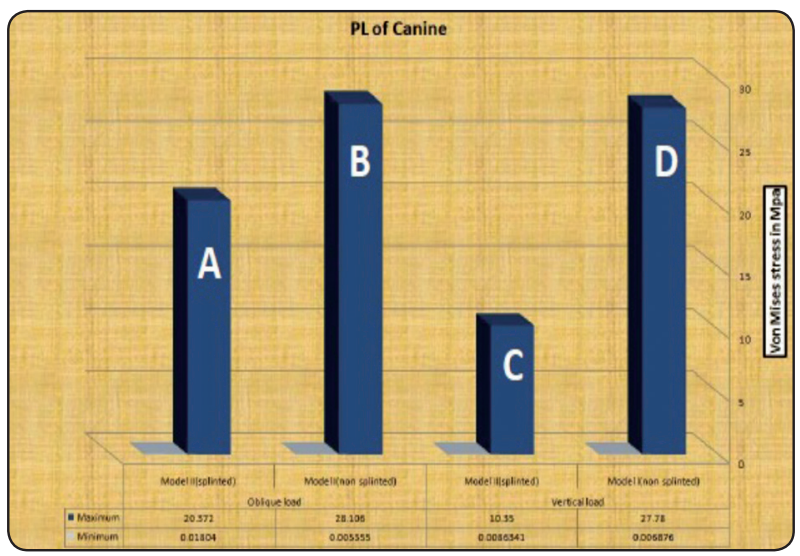

Fig. (6) Bar chart showing maximum stresses recorded in periodontal ligament of loaded side canine in MPa. Oblique loading: A; splinted, B; non-splinted. Vertical loading: C; splinted and D non splinted.

For the implants; the highest stresses were recorded at junction between the loaded implant and crest of the ridge i.e compact bone; at the mesio-buccal side for the two models under the two loading conditions. However the area of stress concentration around the implant for the splinted model was smaller than that of the non-splinted. The values recorded for the splinted model under vertical loading were nearly $\mathbf{5 5 \%}$ of these recorded for the non-splinted. These values were 91.045 $\mathbf{M P a}$ for the non-splinted model and 51.467 MPa for the splinted.

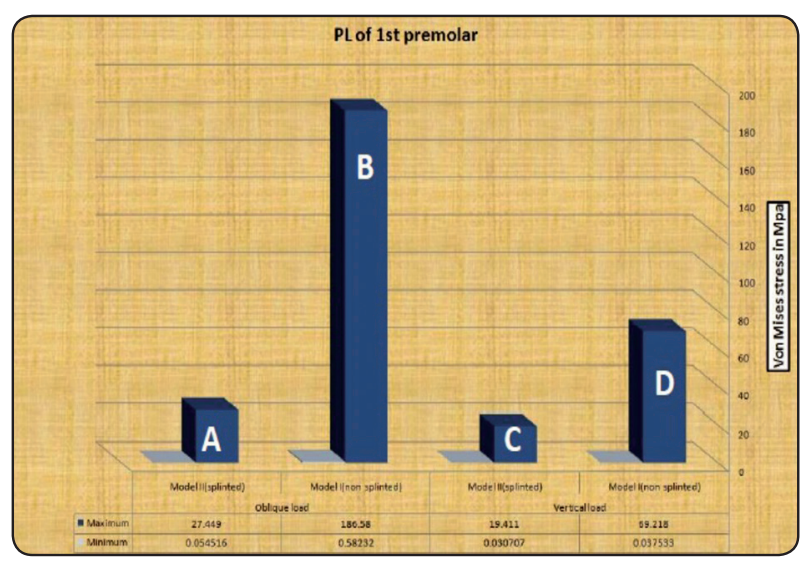

Fig. (5) Bar chart showing maximum stresses generated in the loaded side periodontal ligament of 1 st premolar in MPa. A; splinted, B; non-splinted under oblique loading while $\mathrm{C}$; splinted and D non-splinted under vertical loading

The values recorded for the implant of the splinted model under oblique loading were nearly $110 \%$ of that for the non-splinted. These values were 143.05 MPa for the splinted model and 129.75 $\mathrm{MPa}$ for the non-splinted. In addition the area of stress concentration for the splinted model was greater than that of the non-splinted. Figure $7(A-D)$ shows the stress pattern around the loaded side implants for different loading and splinting conditions.

For the periodontal ligament (PL) of $1 \mathrm{st}$ premolar; the highest stresses were recorded at the disto-lingual side near to the crest of the ridge for all models except the non-splinted model under the vertical load; the stresses were located at the mesio- buccal side. The PL of the 1st premolar in non-splinted model was highly stressed nearly in all locations; while for the splinted condition, the maximum stress recorded was localized to a very small area.

The values recorded for the splinted model under vertical loading were nearly $30 \%$ of these recorded for the non-splinted. These values were $69.218 \mathrm{MPa}$ for the non-splinted model and 19.411 MPa for the splinted. The values recorded for the PL of 1st premolar of the splinted model under oblique loading 


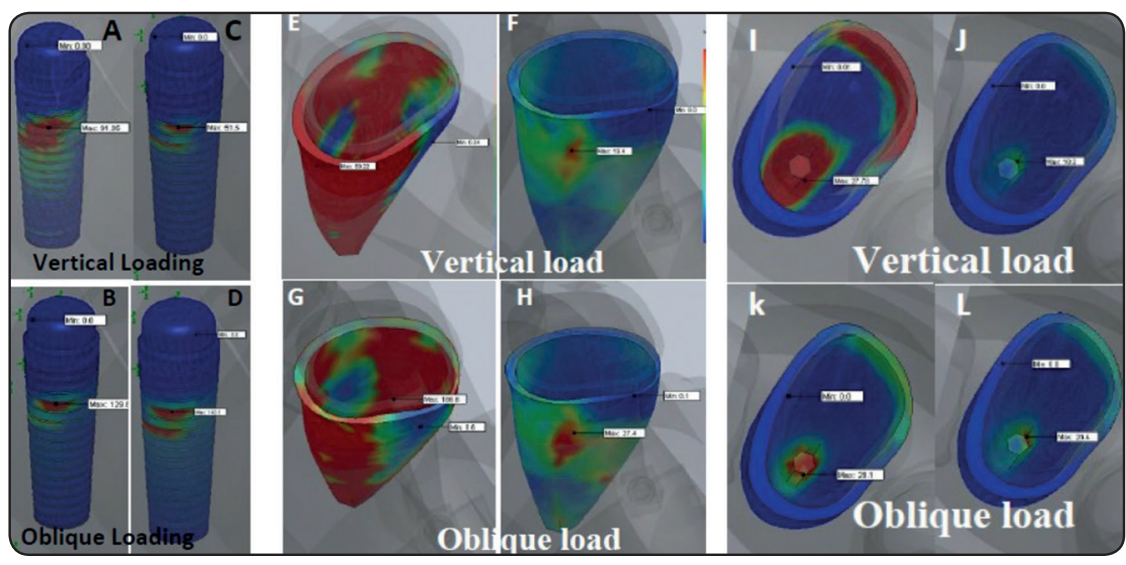

Fig. (7) Stress distribution of loaded side supporting structures under vertical (upper part of figure) and oblique (lower part of figure) loading. Implant in non-splinted (A and B) and splinted (C and D) models. Periodontal ligament of $1^{\text {st }}$ premolar in non-splinted ( $\mathrm{E}$ and $\mathrm{G})$ and splinted (F and $\mathrm{H}$ ) models. Periodontal ligament of canine in non-splinted (I and $\mathrm{K}$ ) and splinted ( $\mathrm{J}$ and $\mathrm{L})$.

were nearly $15 \%$ of that for the non-splinted. These values were 27.449 MPa for the splinted model and $186.58 \mathrm{MPa}$ for the non-splinted.

These values were 27.449 MPa for the splinted model and 186.58 MPa for the non-splinted. Figure $7(\mathrm{E}-\mathrm{H})$ shows the stress pattern around the PL of the 1st premolars for different loading and splinting conditions.

For the PL of canine; the highest stresses were recorded at a localized area at the apex of the root for all models and loading conditions except for the non-splinted model under vertical load, where these stresses were recorded at the apex and a wide area at the disto-buccal side near the crest of the ridge. The stresses generated for the non-splinted model were always higher than those of the splinted and the area of stress concentration during vertical loading was greater than during oblique.

The values recorded for the splinted model under vertical loading were nearly $40 \%$ of these recorded for the non-splinted. These values were 10.35 MPa for the splinted model and 27.78 MPa for the nonsplinted. The values recorded for the PL of canine of the splinted model under oblique loading were nearly $70 \%$ of that for the non-splinted. These values were 20.372 MPa for the splinted model and 28.106 MPa for the non-splinted. Figure 7 (I-L) shows the stress pattern around the PL of canines for different loading and splinting conditions.

\section{For non-loaded side}

The values of maximum stresses generated at the non loading side were very small in general compared to the loading side. Further reduction of the maximum stresses was noticed in case of splinting, and the minimum stress values were 0.00 in all the cases.

There were very low stress values for the PL of the 1st premolars and canines of the contra lateral side in comparison with the loaded side. These values can be omitted as they did not reach $1 \%$ of the loaded side in the average. The only exception was the periodontal ligament of the 1st premolar in case of vertical loading in non-splinted model where this ratio was $3 \%$.

The stresses transmitted to the implants of the non-loaded side were $\mathbf{6 - 1 1 \%}$ of the loaded side for oblique and vertical loading respectively for the nonsplinted model. While for the splinted model; these stresses were only $4 \%$ of that generated for loaded side. The pattern of stress distribution was very homogenous without areas of stress concentration. Table 4 shows the maximum stresses transmitted to these structures. 
TABLE (4) Maximum stress values of implants and periodontal ligaments for non-loaded side.

\begin{tabular}{|l|c|c|c|c|}
\hline \multirow{2}{*}{} & \multicolumn{2}{|c|}{ Vertical load } & \multicolumn{2}{c|}{ Oblique load } \\
\cline { 2 - 5 } & Splinted & Non splinted & Splinted & Non splinted \\
\hline Implant & 2.0678 & 10.264 & 5.0008 & 8.4897 \\
\hline PL/PM & 0.046171 & 2.122 & 0.038145 & 0.76762 \\
\hline PL/C & 0.038454 & 0.6577 & 0.15539 & 1.4119 \\
\hline
\end{tabular}

PL/PM; periodontal ligament of first premolar.

\section{$P L / C$; periodontal ligament of canine.}

\section{DISCUSSION}

In the present study, 3-D FEA was used to evaluate the effect of splinting of two abutments on stress distribution around abutment teeth and implants. These implants supporting mandibular distal extension base RPD retained by extra coronal attachments.

To obtain more reliable and indicative results; the model was idealized as much as possible. The term idealization refers to how much the simulation imitates the real clinical situation. To achieve idealization; a CT scan data of a middle age patient was used to construct the model. This technique is very useful to identify the quantitative and qualitative differences between various structures which facilitated the production of an accurate model ${ }^{11}$. This patient had no amalgam fillings to avoid artifacts and no developmental abnormalities, gross defects, or previous surgery to avoid boney defects at areas of interest.

Another step toward idealization was the use of MIMICS 10.01 software. This software program can differentiate between different anatomical parts based on their radio-opacity. Due to some difficulties encountered in the present study; complete production of the model by the program was not possible. This was due to inability to identify the model as a solid body when it was exported to Solidworks software.
A possible solution for completed production of the model by the previously mentioned software programs was to import the file into reverse engineering software. Such software is usually used to smooth, flatten and fill the small surface holes of the model to achieve more simplified unirregular model ${ }^{8}$. Because such software was not available a decision was made to draw the mandible in Solidworks software guided by its coronal cut images. The teeth; on the other hand; were exported directly to Solidworks software may be because of its small size files or their less complexity.

The thickness of mucosa and microstructures of bone at different locations were modeled in accordance with relevant literatures ${ }^{7,8}$. Teeth were also drawn as a result of complicated meshing due to root canal space, while periodontal ligaments were drawn due to difficulties in importing them by the software. Drawing of the periodontal ligaments was similar to the work of Wang et al (2011). ${ }^{9}$ Simulation of the periodontal ligament in FEA models is a recent modification of the technique and opens the way for understanding the stresses created in the natural abutment in a better way.

The selected implant position was based on the recommendations suggesting that the implant should be placed at the area of second molar in order to effectively change the class I situation to a class III ${ }^{7}$.

All components of this study were subtracted from each other leaving one component with reference points by which assembly could be done. This procedure was carried out to simplify component assembly and material properties identification by the software.

Only one model of the distally extension edentulous mandible was constructed and two copies were made for the non-splinted and splinted situations. After that each completed model was copied to create two identical loading models "oblique and vertical load". These procedures eliminated any possible variation between the 
loading and splinting conditions as a result of geometry changes.

The limitations of the current study are that all materials of the model were considered to be homogenous, isotropic and linearly elastic. Such an assumption is not real and ignores the anisotropic and non linear behavior of the supporting structures. Moreover the response of bone including repair capacity or failure limit as well as ligamentous and muscular attachments were not simulated. All these factors would affect complete interpretation of the results. However the comparative nature of the present study "between different designs and loading conditions" reduces the significance of the previously mentioned factors.

The load directions used in the present study represent a more realistic condition. Occlusal forces are not always axial or horizontal, but more likely is oblique in nature as a result of these combinations. Therefore the oblique load application was selected and the vertical was used as a reference.

The curvature-based mesh was used for the current study rather than standard mesh. The curvature-based mesh supports compatible meshing between touching solid faces. It can also check for interference between bodies before meshing. If interferences are detected, meshing stops and the interference detection property manager views the interfering parts and resolves all interferences before meshing again.

The results of the present study demonstrated several tendencies. Splinting reduces stresses under vertical loading. This is consistent with the findings of Nishimura et al (1999) ${ }^{14}$, $\mathrm{Ku}$ et al (2000) ${ }^{15}$, Awang et al $(2008)^{3}$, Chikunov et al $(2008)^{4}$, and Wang et al (2011) ${ }^{9}$.

The effect of implant placement is evidenced also by its great load sharing in every design and load conditions tested. The highest stresses generated in all the supporting structures were always registered at the bone implant interface. The effect of implant placement is more important in cases of non-splinted model as it shares greater stresses than splinted designs. This fact also indicated that splinting synergizes implant placement and must be a basic design feature if a single implant is inserted at the distal area and the RPD is retained by a resilient attachment.

The effect of implant placement is more important during oblique loading as it prevent horizontal displacement at its posterior end. This is evident by the great reduction of stresses generated in the mucosa. This stress reduction was evident between vertical and oblique loading either for splinted or non-splinted designs. This may lead to less bone resorption, less need for rebasing and less tension for attachments $6,7,13$.

A general tendency found in the present study is that oblique loading produces higher stress concentrations than vertical in all models. Comparing the non-splinted models reveals that oblique loading tend to double, triple or even produces ten folds more stresses than vertical loading the same structures. Oblique loads have the greatest effect on the terminal abutments, implants and supporting structures in comparison with vertical loads

Oblique loading produces more complex denture base movement. This movement will create multidirectional stresses in the supporting structures. Part of this movement will be resisted by the implant at the distal end. Another part will be resisted by the abutment tooth. Splinting the abutments improves its resistance to movement and load sharing. The rigidity of the partial denture and its extension to the other side add more to resistance to oblique loading. The end effect will be increased resistance to displacement of the denture base hence the increased stresses transmitted to the supporting structures of the loaded side and decreased stresses to non-loaded side. On the other hand this led to the decreased stresses registered at the non-loaded side which were generally very small.

This denture base movement is increased in nonsplinted model hence produces more stresses at the 
non-loaded side and highlights the importance of cross-arch retention and stabilization. The primary movement of the abutment is always at the cervical region and the secondary movement is always at the apex and fulcrum point lies somewhere in between. A secondary effect would be; the transmission of indirect stresses to the neighboring abutments as seen in the periodontal ligament of the canine in case of non-splinted models. Splinting in this case produces more homogenous distribution of stresses without areas of great concentrations.

The design that produces less movement at the cervical area of the abutment and apical area would be the design of choice. In view of the results of the present study this design will be splinted abutments with vertical loading i.e. reduced cusp angles.

\section{CONCLUSION}

Within the limitations of this study, the following conclusions could be drawn:

1. Simulation of the periodontal ligament is beneficial in viewing the stress pattern around the abutment teeth.

2. Splinting the primary abutment to the adjacent tooth produces more homogenous distribution of stresses at the supporting structures without areas of great concentrations. So it would be the design of choice even with using an implant to support the distal extension RPD.

3. Reducing the amount of oblique loading in these designs (i.e. less cusp angle) would be and advantage.

\section{RECOMMENDATIONS}

Further clinical researches preferably long-term clinical randomized controlled studies are needed to reach a final conclusion regarding the use of implant supported RPD synergized with extra-coronal attachments.

\section{CLINICAL SIGNIFICANCE}

The clinical significance of present study is: a cost effective use of implants to convert a free end prosthesis to one that is tooth-implant supported retained by extra coronal attachments. This is also useful biomechanically as load on the abutment teeth was reduced and other structures were uniformly loaded.

The old design principle of reducing the cusp angle is still valid in this case, so it is beneficial to reduce the cusp angle of the implant supported RPD. This will decrease the lateral component of load and direct most of the transmitted load vertically to the abutment teeth.

\section{REFERENCES}

1. Costa MM, da Silva MA, Oliveira SA, Gomes VL, Carvalho PM, Lucas BL.: Photoelastic study of the support structures of distal-extension removable partial dentures. J . Prosthodont. 2009: 18; 589-595.

2. Takayuki, K. Noriyuki, W.: Influence of molar support loss on stress and strain in premolar periodontium: A patientspecific FEM study. J. Dent. 2009; 37 : 541 - 548

3. Awang RAR, Arief EM, Hassan A.: Spring loaded plunger attachment for retention of removable partial denture: a case report. Arch Orofac Sci 2008;3: 32-35.

4. Chikunov I, Doan P, Vahidi F. Implant-retained partial overdenture with resilient attachments. J Prosthodont. 2008; 17(2): 141-148.

5. El-Chsrkawy H. G., El Wakad M. T. : Effect of splinting on load distribution of extracoronal attachment with distal extension prosthesis in vitro. J.Prosthet. Dent. 1996;76:315-320

6. Naert. I., Koutsikakis, G., Quirynen, M., Jacolbs, R., Van Steenberghe, and D.: Biologic outcome of implant supported restoration of partial edentulism (part I). Cin. Oral Impl. Res. 2002; 13:381.

7. Ohkubo C, Kurihara D, Shimpo H, Suzuki Y, Kokubo Y, Hosoi T.: Effect of implant support on distal extension removable partial dentures: in vitro assessment. J Oral Rehabil. 2007; 34(1): 52-56. 
8. Daas M., Dubois G., Bonnet A.S., Lipinski P., RignonBret C. : A complete finite element model of a mandibular implant-retained overdenture with two implants: Comparison between rigid and resilient attachment configurations. Medical Engineering \& Physics. 2008; 30: 218-225.

9. Wang H.Y., Zhang Y.M., Yao D., Chen J.H.: Effects of rigid and non-rigid extra-coronal attachments on supporting tissues in extension base partial removable dental prostheses: a nonlinear finite element study. J. Prosthet. Dent. 2011; 105(5): 338-346.

10. Cağlar A, Aydin C, Ozen J, Yilmaz C, Korkmaz T.: Effects of mesiodistal inclination of implants on stress distribution in implant-supported fixed prostheses. Int J Oral Maxillofac Implants. 2006 Jan-Feb;21(1):36-44.

11. Geramy A., Adibrad M., Sahabi M.: The effects of splinting periodontally compromised removable partial denture abutments on bone stresses: a three-dimensional finite element study. J. Dent. Sci. $2010 ; 5(1)$ : 1-7.

12. Jian-Ping G., Keson B.C. Tan, Gui-Rong L.: Application of finite element analysis in implant dentistry: A review of the literature. J Prosthet Dent 2001 85;585-598.

13. Halterman SM, Rivers JA, Keith JD, Nelson DR. Implant support for removable partial overdentures: a case report. Implant Dent. 1999;8:74-78.

14. Nishimura RD, Ochiai KT, Caputo AA, Jeong CM. Photoelastic stress analysis of load transfer to implants and natural teeth comparing rigid and semirigid connectors. J Prosthet Dent 1999; 81:696-703.

15. Ku Y, Shen F, Chan C. Extracoronal resilient attachments in distal extension removable partial dentures. Quintessence Int. 2000; 31(5): 311. PMID: 11203941 